\title{
Giant impact stratification of the Martian core
}

\author{
Jafar Arkani-Hamed ${ }^{1}$ and Peter Olson ${ }^{2}$ \\ Received 19 October 2009; revised 6 December 2009; accepted 17 December 2009; published 20 January 2010.
}

[1] We investigate the direct thermal effects of giant impacts on the Martian core and its dynamo. Shock wave heating of Mars is calculated in terms of the impact velocity and the final basin size. Although much of the shock wave heat is deposited in the mantle, shock heating from a giant impact produces non-uniform temperatures in the core, leading to an overturn event and stable thermal stratification in the liquid core. Numerical dynamos with core heating from polar and equatorial impacts show that the overturn and stratification quickly destroys a pre-existing core dynamo, within ten thousand years. Energy considerations reveal that both the stratification and the time required for removal of the stratification increase with impact size. Our calculations indicate that several tens to over one hundred million years are required for removal of core stratification following a giant impact. Citation: Arkani-Hamed, J., and P. Olson (2010), Giant impact stratification of the Martian core, Geophys. Res. Lett., 37, L02201, doi:10.1029/2009GL041417.

\section{Introduction}

[2] Although Mars lacks a global magnetic field at present, the observed magnetic anomalies imply a core dynamo that diminished in strength around $\sim 4$ Ga [e.g., Arkani-Hamed, 2004; Lillis et al., 2008]. Many scenarios have been proposed to explain the cessation of the Martian dynamo, including core solidification [Young and Schubert, 1974], an early end to plate tectonics [Nimmo and Stevenson, 2000], impact heating of the mantle [Roberts et al., 2009], perturbation of an already subcritical dynamo [Kuang et al., 2008], and satellite in-fall [Arkani-Hamed, 2009].

[3] A progressive decrease in the strength of the Mars crust magnetic field overlaps the formation time of about 20 giant impact basins [Frey, 2008]. Roberts et al. [2009] showed how impact heating in the mantle alters the heat loss from the core, which could indirectly suppress a convective dynamo. Here we show that shock waves from giant impacts directly but differentially heat the core, resulting in thermal stratification of the liquid core within a few hundreds of years, magnetic field suppression on the time scale of ten thousand years, and inhibition of convection lasting millions of years.

\section{Impact Heating of the Martian Core}

[4] Impact-induced temperature perturbations in Mars interior can be related to basin diameter and impact velocity

\footnotetext{
${ }^{1}$ Department of Physics, University of Toronto, Toronto, Ontario, Canada.

${ }^{2}$ Earth and Planetary Sciences, Johns Hopkins University, Baltimore, Maryland, USA.

Copyright 2010 by the American Geophysical Union. 0094-8276/10/2009GL041417\$05.00
}

by combining standard results from impact theory and shock wave propagation. The basin transient diameter $D_{t r}$ is related to its final diameter $D_{b}$ by

$$
D_{b}=1.02 D_{*}^{-0.086} D_{t r}{ }^{1.086}
$$

[Holsapple, 1993], which is related to the diameter of the impacting body $D_{\text {imp }}$ by

$$
D_{i m p}=0.69 D_{t r}^{1.27} U^{-0.56} g_{s}^{0.22}
$$

[Melosh, 1989], where $D_{*}$ is the transition diameter from simple to complex crater ( $7 \mathrm{~km}$ for Mars), $U$ is the impact velocity in $\mathrm{km} / \mathrm{s}$, and $g_{s}$ is the gravitational acceleration at the surface. Shock wave pressure $P_{s}$ versus distance $r$ from the center of the isobaric impact sphere is given by [Pierazzo et al., 1997]

$$
P_{s}=\rho_{o}\left(C+S u_{p}\right) u_{p} \quad r<r_{c}
$$

$$
P_{s}=P_{c}\left(r_{c} / r\right)^{n o} \quad r>r_{c} ; n_{o}=-1.84+2.61 \log U
$$

where $\rho_{o}$ and $C$ are the pre-shocked density and acoustic velocity, $u_{p}$ is the particle velocity (= one half the impact velocity, assuming similar target and impacting materials), $S$ is a constant, and $r_{c}\left(=0.225 D_{i m p} \mathrm{U}^{0.211}\right)$ is the isobaric sphere radius, approximately the depth of penetration of the impacting body. Parameters in equations (3a) and (3b) are in SI units, except for $U$ which is in $\mathrm{km} / \mathrm{s}$. The irreversible temperature increase $\Delta T$ due to the pressure disturbance is determined using both ordinary and foundering models by Watters et al. [2009]

$$
\Delta T=P_{\delta}(1-1 / f) /\left(2 \rho_{o} S\right)-(C / S)^{2}(f-\ln f-1),
$$

where

$$
\begin{gathered}
\left.f=-P_{\delta} /\left(\beta\left\{1-\left[\left(2 P_{\delta} / \beta\right)+1\right]^{1 / 2}\right]\right\}\right) \\
P_{\delta}=P_{\mathrm{s}}-P_{\mathrm{o}}
\end{gathered}
$$

and

$$
\beta=\left(C^{2} \rho_{o}\right) /(2 S)
$$

[5] The lithostatic pressure $P_{\mathrm{o}}$ is calculated using a Mars model consisting of a uniform liquid core and an overlying uniform mantle, with parameter values listed in Table 1. The $1700 \mathrm{~km}$ core radius is within the range of recent estimates, $1520-1840 \mathrm{~km}$ [Yoder et al., 2003]. The shock wave focus 
Table 1. Physical Parameters Used in the Models

\begin{tabular}{lc}
\hline \multicolumn{1}{c}{ Parameter } & Unit \\
\hline$R=$ Mars radius & $3390(\mathrm{~km})$ \\
$R_{c}=$ Core radius & $1700(\mathrm{~km})$ \\
$g_{s}=$ Gravity at the surface & $3.72\left(\mathrm{~m} / \mathrm{s}^{2}\right)$ \\
$g_{c m b}=$ Gravity at core mantle boundary $(\mathrm{cmb})$ & $2.93\left(\mathrm{~m} / \mathrm{s}^{2}\right)$ \\
$\rho_{i}=$ Impactor density & $3000\left(\mathrm{~kg} / \mathrm{m}^{3}\right)$ \\
$\rho_{m}=$ Mantle density & $3500\left(\mathrm{~kg} / \mathrm{m}^{3}\right)$ \\
$\rho_{c}=$ Core density & $7500\left(\mathrm{~kg} / \mathrm{m}^{3}\right)$ \\
$C_{p m}=$ Specific heat of the mantle & $1200(\mathrm{~J} / \mathrm{kg} / \mathrm{K})$ \\
$C_{p c}=$ Specific heat of the core & $600(\mathrm{~J} / \mathrm{kg} / \mathrm{K})$ \\
$\alpha=$ Thermal expansion coefficient of the core & $10^{-5}(1 / \mathrm{K})$ \\
$\eta=$ Magnetic diffusivity of the core & $1.0(\mathrm{~m} / \mathrm{s})$ \\
$K=$ Thermal conductivity of the core & $40(\mathrm{~W} / \mathrm{m} / \mathrm{K})$ \\
$\kappa=$ Thermal diffusivity of the core & $9.5 \times 10^{-6}\left(\mathrm{~m}^{2} / \mathrm{s}\right)$ \\
$\Omega=$ Angular velocity of the core rotation & $7 \times 10^{-5}(\mathrm{rad} / \mathrm{s})$ \\
$R_{m c}=$ Critical magnetic Reynolds number of the core & 40 \\
$q_{c m b}=$ Convective heat flow at cmb & $0.040\left(\mathrm{~W} / \mathrm{m}^{2}\right)$ for super-giant \\
$F=$ Convective buoyancy flux below cmb $\left(\alpha g_{c m b} q_{c m b} / \rho_{c} C_{p c}\right)$ & $2.44 \times 10^{-13}\left(\mathrm{~m}^{2} / \mathrm{s}^{3}\right)$ \\
$U=$ impactor velocity & 10 and $15(\mathrm{~km} / \mathrm{s})$ \\
$E i=$ Kinetic energy of the impact & $0.015\left(\mathrm{~W} / \mathrm{m}^{2}\right)$ for giant impact, \\
& $1.4 \times 10^{28} \mathrm{~J}$ for giant impact $(3000 \mathrm{~km} \mathrm{basin}) ; 1.6 \times 10^{29} \mathrm{~J}$ \\
C $=$ acoustic velocity & for super-giant impact $(6000 \mathrm{~km}$ basin $)$ \\
$\mathrm{S}=$ the constant in the scaling law & $7.24 \mathrm{~km} / \mathrm{s}$ for mantle, $4 \mathrm{~km} / \mathrm{s} \mathrm{for} \mathrm{core}$ \\
\hline
\end{tabular}

is taken as the center of the isobaric core of the impact. Shock wave amplitude in the core is calculated using seismic ray theory following Aki and Richards [2002]. We include effects of near-surface reflection plus reflection and transmission of the direct wave at the core-mantle boundary $(\mathrm{cmb})$. We do not consider interference of the reflected wave with the direct wave on the far side of the core, because the pressure disturbance is small there.

[6] Five of the largest giant impact basins on Mars have diameters between 2640 and $3380 \mathrm{~km}$ [Frey, 2008]. Figure 1 shows the shock-induced temperature increase in the core for a representative basin of diameter $3000 \mathrm{~km}$, an impact velocity of $U=15 \mathrm{~km} / \mathrm{s}$, and the ordinary shock model, where $P_{\mathrm{o}}$ is set to zero. The decrease in wave speed as it enters the liquid core produces a distinct shadow zone (black region) where the shock wave does not penetrate. Less than $2 \%$ of the kinetic energy of the impacting body $\left(1.4 \times 10^{28} \mathrm{~J}\right)$ heats the core, yet the resulting temperature perturbations are relatively large, due partly to the low specific heat of liquid iron. Particularly below the impact site, they far exceed the non-adiabatic temperature variations associated with convection in iron-rich liquid cores, which are estimated to be of order 0.1 K [Stevenson, 1987].

\section{Core Stratification}

[7] In response to the temperature perturbations shown in Figure 1, the liquid core experiences a thermally-driven overturn event that re-distributes the shock-heated liquid, creating a stable stratification. Assuming the core was initially close to a well-mixed state, the resulting stable thermal stratification can be calculated by adiabatic re-filling of the core according to temperature. Figure 2 shows the volume fraction of the core versus the shock-heated temperature anomaly (PDF), the shock-heated temperature anomaly profile through the core along the axis of the impact prior to overturn, the radial profile of re-distributed temperature after re-filling, and the corresponding buoyancy frequency $N=$ $(\alpha g d T / d r)^{1 / 2}$, where $T(r)$ is the re-distributed temperature relative to the adiabat, $g(r)$ is acceleration of gravity, and $\alpha$ is thermal expansion coefficient. The strength of the stable stratification is proportional to $N$. Also shown in Figure 2 are the results for a $15 \mathrm{~km} / \mathrm{s}$ impact producing a $6000 \mathrm{~km}$ diameter basin, as has been previously proposed for creating the northern lowland [Andrews-Hanna et al., 2008]. Note that the core stratification in Figure 2 increases with impact size, from $\sim 0.38$ of the core volume for the smaller impact to 0.47 for the larger one.

\section{Dynamo Response to Shock Heating of the Core}

[8] We model the short-term response of the Martian dynamo to shock heating of its core with numerical dynamos.

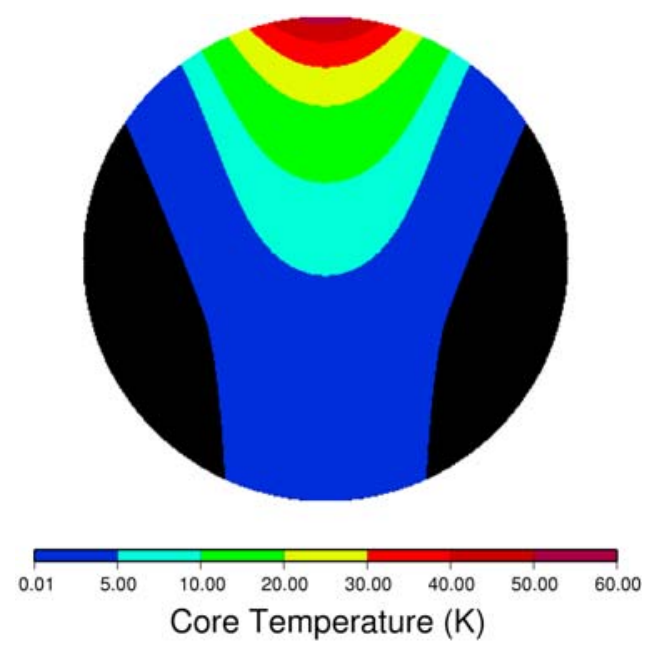

Figure 1. Axisymmetric shock wave-induced temperature perturbations in the core due to an impact creating a $3000 \mathrm{~km}$ diameter basin on Mars surface. The sub-impact point on the core-mantle boundary is at the top of the figure. The region with negligible shock heating (black color) occupies about 0.38 of the core volume. Calculation parameters are given in Table 1. 

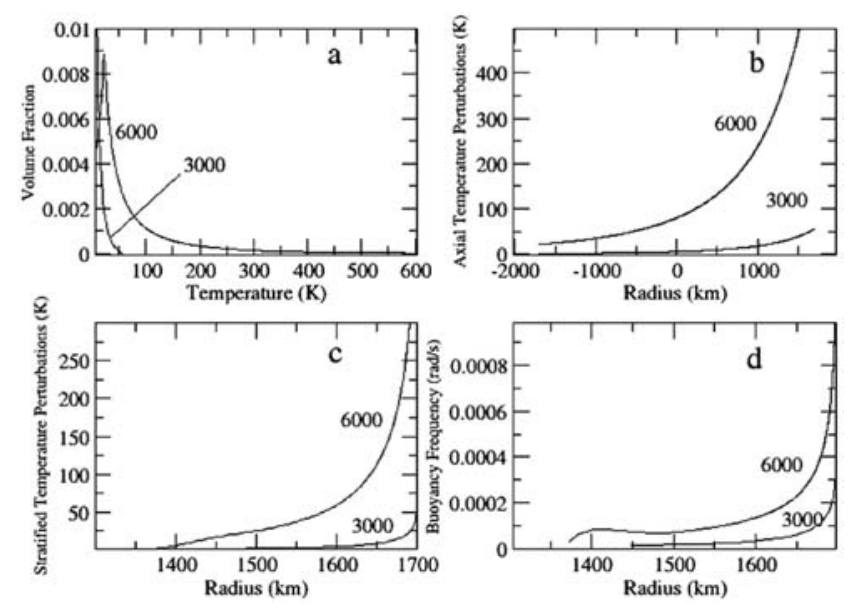

Figure 2. Temperature perturbation and stratification following impact-induced shock heating in the Martian core. (a) Volume fraction of the liquid core versus shockinduced temperature perturbation (PDF); (b) temperature perturbation along the axis of the impact prior to stratification, with (positive, negative) radii corresponding to (near, far) side of the core; (c) radial profile of temperature perturbation after adiabatic stratification; (d) radial profile of buoyancy frequency $\mathrm{N}$ after adiabatic stratification. 6000, 3000 denote final basin diameters in $\mathrm{km}$.

The base model is an equilibrated numerical dynamo with parameter values $E=1 \times 10^{-3}, P r=1$, and $P m=5$ (where $E$, $P r$, and $P m$ are Ekman, Prandtl, and magnetic Prandtl numbers, respectively), a Rayleigh number $R a$ five times critical, and uniform $\mathrm{cmb}$ heat flow. The definitions of these dimensionless parameters are given by Christensen and Wicht [2007]. Although the core is completely molten in the impact heating and thermal evolution calculations, a small solid inner core is added to avoid singularities at $r=$ 0 of the dynamo equations. We superimpose on the base model axi-symmetric thermal perturbations with the pattern shown in Figure 1. Two impact locations are examined, one at the equator, the other at the north pole. Because of numerical constraints, the ratio of the maximum temperature difference in the perturbations to the maximum temperature difference in the base model is $\boldsymbol{\theta}=30$, whereas Figure 2 indicates that $\boldsymbol{\theta}>50$ is more realistic for giant impacts on Mars. We expect that the dynamo perturbation following a $\boldsymbol{\theta}>50$ impact would be greater than the ones considered here.

[9] Figure 3 shows short-term dynamo responses to these two thermal perturbations. The images are snapshots of temperature (base + perturbation) in the equatorial plane of the core at the numbered time steps following impact, one time step corresponding to about 8 yrs according to the parameters in Table 1.

[10] In response to the equatorial impact (Figure 3a), shock-heated fluid from the sub-impact region spreads out beneath the $\mathrm{cmb}$, while the less-heated fluid sinks toward the inner core. The response to the polar impact is more complex. As seen in steps 15 and 50 of Figure 3b, the shock-heated fluid deep in the core breaks up into thermal Rossby waves and migrates toward the $\mathrm{cmb}$. Meanwhile, shock-heated fluid at shallower levels in the core spreads out beneath the $\mathrm{cmb}$ from the sub-impact point, reaching the equatorial plane between steps 50 and 200 in Figure $3 \mathrm{~b}$. In both cases, the rapid overturn events lead to stable stratification, although at slightly different rates.

[11] Following the equatorial impact, the overturn event displaced the magnetic dipole axis toward the far side of the core. Following the north polar impact, the overturn event involved generally north to south flow beneath the $\mathrm{cmb}$, which transported magnetic field lines southward. In both scenarios, the field intensity is reduced in the sub-impact region relative to the far side.

[12] Figure 4 shows the evolution of kinetic and magnetic energy in these two dynamos relative to their base levels versus time following impact, in dimensionless units of the dipole free decay time. One Mars dipole free decay time is $\left(\mathrm{R}_{\mathrm{c}} / \boldsymbol{\pi}\right)^{2} / \eta \sim 9.3 \mathrm{kyr}$, according to Table 1 . As shown in Figure 4, both impacts produce transient increases in kinetic and magnetic energy during overturn, but the stratification quenches the convection within a few hundred years, and thereafter the magnetic energy decreases by more than an order of magnitude within one dipole decay time.

[13] By inhibiting radial fluid motions, stable stratification works against any dynamo mechanism, but it is especially potent if the early Mars dynamo was maintained by thermal convection. A mechanically or chemically-driven dynamo might survive the impact heating, but removal of the stratification is a prerequisite for a thermal convection dynamo, and this requires significant amounts of time. In the dynamo models shown in Figures 3 and 4, the stable (a)
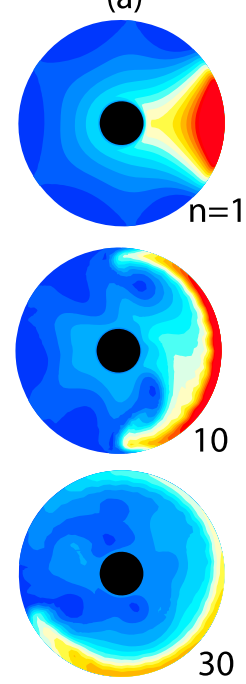

30

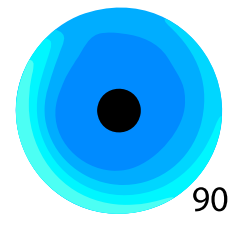

(b)
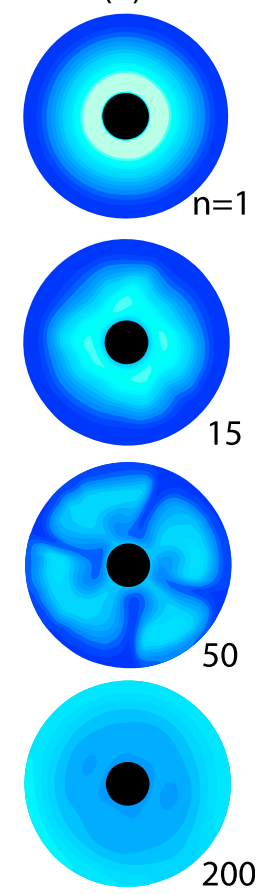

Figure 3. Numerical dynamo models of shock-induced heating of the Martian core by giant impacts. Images show contours of dimensionless temperature perturbation (normalized by $\theta$ ) in the equatorial plane of the core following shock heating, at the time steps indicated by n. One time step is $\sim 8$ yrs for Mars core. (a) Equatorial impact; (b) north polar impact. Contour values of dimensionless temperature perturbation increase from blue $=0$ to red $=1$. The black center shows the inner core. 

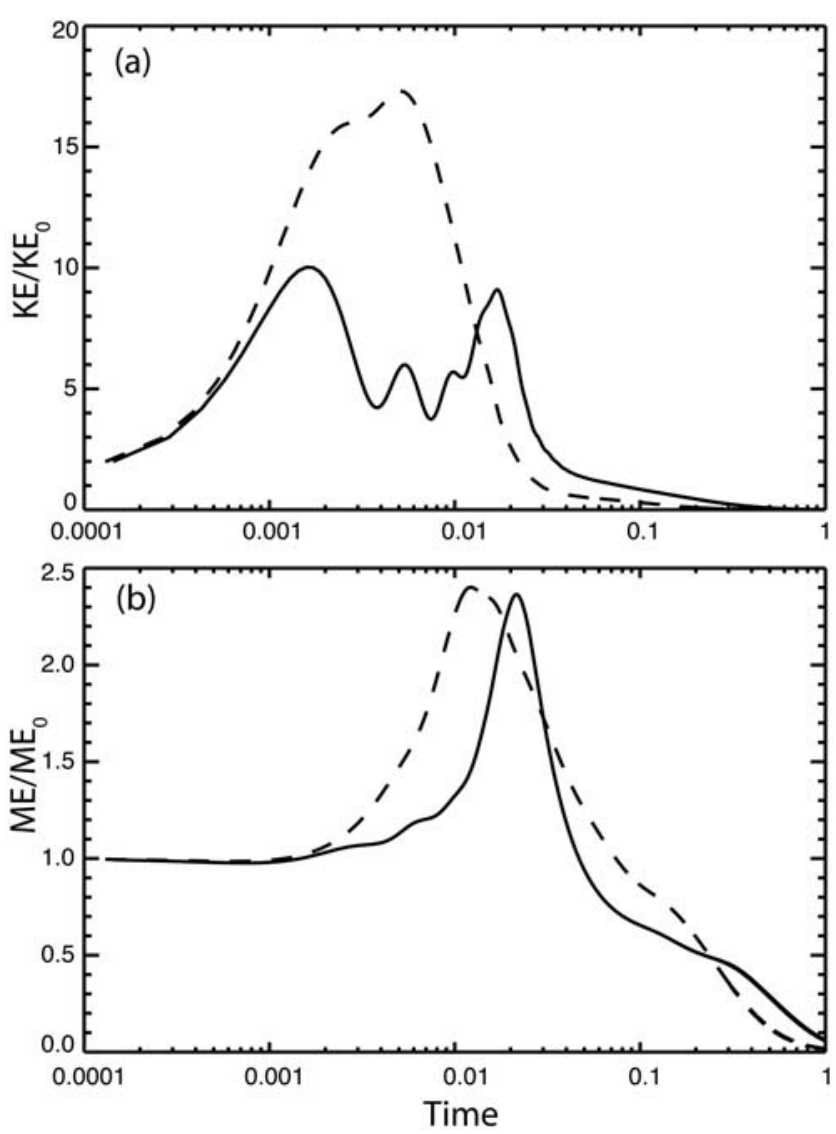

Figure 4. The quenching of numerical dynamos in Figure 3 following shock-induced heating of their cores. (a) Kinetic energy relative to base (pre-shock) kinetic energy; (b) Dynamo magnetic energy relative to base (pre-shock) magnetic energy. Dashed curves denote equatorial impact (Figure 3a), solid curves denote north polar impact (Figure $3 b$ ). Time is in dimensionless units of dipole free decay. One dipole free decay time is approximately $9.3 \mathrm{kyr}$ for Mars.

stratification is eventually removed by thermal diffusion. For Mars, a simple estimate of the time to remove the stratification is the ratio of heat added to the core at impact to the base rate of core heat loss, which have been calculated to be about $15 \mathrm{~mW} / \mathrm{m}^{2}$ at the time of the giant impact sequence [Roberts et al., 2009] and about $40 \mathrm{~mW} / \mathrm{m}^{2}$ when the lowlands were formed [e.g., Williams and Nimmo, 2004]. For 3000 and $6000 \mathrm{~km}$ diameter basin impacts, these heat loss rates yield stratification removal times of $13 \mathrm{Myr}$ and $66 \mathrm{Myr}$, respectively.

[14] The radial temperature profiles in the core shortly after the overturn event consists of a strongly stratified region in the outer part of the core, a weaker stratified layer in the middle, and essentially adiabatic temperature in deeper part up to $\sim 1450 \mathrm{~km}$ radius, as shown in Figure 2 . The adiabatic inner part will not convect because it is overlain by a hotter layer relative to the adiabatic temperature at $\sim 1450 \mathrm{~km}$ radius. The middle layer will not convect because of positive temperature gradient. Convection gradually develops in the upper parts of the highly stratified region and penetrates down in time until all of the impact heat is exhausted. We estimate how much of this stratification must be removed in order to establish convective dynamo conditions, as follows. Self-sustaining dynamo action requires that the magnetic Reynolds number $R_{m}=w z / \eta$, where $w$ is fluid velocity in a layer of thickness $z$, exceed a critical value $R_{m c} \sim 40$ [Christensen and Wicht, 2007]. Rotating convection experiments indicate fluid velocities scale like $w \sim(F / \Omega)^{1 / 2}$, where $F$ and $\Omega$ are buoyancy flux in the fluid and planetary rotation rate, respectively [Levy and Fernando, 2002]. Eliminating $w$ from these relations, we find that self-sustaining dynamo conditions are reached when convection extends through a layer of thickness $z_{c} \sim \eta R_{m c}$ $(\Omega / F)^{1 / 2}$. Using Table 1 values, we find $z_{c}>700 \mathrm{~km}$ for Mars, which is thicker than the thermally stratified region in the core associated with a $3000 \mathrm{~km}$ impact basin (see Figure 2). Therefore, nearly all of the impact heat must be removed from the core before convective dynamo conditions are reached.

\section{Discussion}

[15] Our calculations show that an impact capable of creating a basin $3000 \mathrm{~km}$ in diameter differentially heats the Martian core, with approximately $+60 \mathrm{~K}$ temperature increase below the $\mathrm{cmb}$ at the sub-impact point. Our calculations are based on the same impact heating model used for Mars mantle by Roberts et al. [2009]. We show in Table 2 a suite of results for both ordinary and foundering shocks, impact velocities of 10 and $15 \mathrm{~km} / \mathrm{s}$ creating basins of 3000 and $6000 \mathrm{~km}$ diameters, as well as for the $3380 \mathrm{~km}$ diameter Utopia basin. All result in significant core heating and stratification except for the foundering small impact case, consistent with Watters et al. [2009], who showed that foundering models yield relatively small impact temperature perturbations.

[16] Direct impact-induced temperature perturbations overturn and stratify the liquid core, inhibiting dynamo action and suppressing a pre-existing magnetic field within $\sim 10 \mathrm{Kyr}$, according to our dynamo models. We estimate that it takes about 13 Myr to remove this stratification by core heat loss. Following a super-giant impact creating a $6000 \mathrm{~km}$ basin, comparable to the size of the northern lowlands on Mars [Andrews-Hanna et al., 2008], as much as $66 \mathrm{Myr}$ is needed. We also note that, because direct heating of the core by successive impacts is a cumulative process, the sequence of giant impacts around $\sim 4$ Ga might have a greater

Table 2. Impact Heating Models ${ }^{\mathrm{a}}$

\begin{tabular}{ccccc}
\hline $\mathrm{D}_{\mathrm{b}}$ & $\mathrm{U}$ & Shock & $\mathrm{S}$ & $\tau$ \\
\hline 3000 & 10 & $\mathrm{O}$ & yes & 85.6 \\
3000 & 10 & $\mathrm{~F}$ & yes? & 1.0 \\
3000 & 15 & $\mathrm{O}$ & yes & 21.0 \\
3000 & 15 & $\mathrm{~F}$ & no & 0.0 \\
3380 & 10 & $\mathrm{O}$ & yes & 110.8 \\
3380 & 10 & $\mathrm{~F}$ & yes & 2.4 \\
3380 & 15 & $\mathrm{O}$ & yes & 13.1 \\
3380 & 15 & $\mathrm{~F}$ & yes? & 0.03 \\
6000 & 10 & $\mathrm{O}$ & yes & 148.1 \\
6000 & 10 & $\mathrm{~F}$ & yes & 18.6 \\
6000 & 15 & $\mathrm{O}$ & yes & 65.6 \\
6000 & 15 & $\mathrm{~F}$ & yes & 6.8 \\
\hline
\end{tabular}

${ }^{\mathrm{a}} \mathrm{D}_{\mathrm{b}}$ is basin diameter $(\mathrm{km}), \mathrm{U}$ is impact velocity $(\mathrm{km} / \mathrm{s}), \mathrm{O}$ is ordinary shock and $\mathrm{F}$ is foundering shock, $\mathrm{S}$ is the core stratification, and $\tau$ is the removal time of the impact heat (Myr). In models with "?" the impact heats only the upper most part of the core in the sub-impact region. 
affect on the core stratification than the isolated impact events considered here.

[17] Acknowledgments. This research is partly supported by Natural Sciences and Engineering Research Council (NSERC) of Canada to JAH, and partly by grant EAR-0604974 from the US National Science Foundation to PO.

\section{References}

Aki, K., and P. G. Richards (2002), Quantitative Seismology, 2nd ed., Univ. Sci., Sausalito, Calif.

Andrews-Hanna, J. C., M. T. Zuber, and B. Banerdt (2008), The Borealis basin and the origin of the Martian crustal dichotomy, Nature, 453, 1212-1216, doi: 10.1038 /nature 07011 .

Arkani-Hamed, J. (2004), Timing of the Martian core dynamo, J. Geophys. Res., 109, E03006, doi:10.1029/2003JE002195.

Arkani-Hamed, J. (2009), Did tidal deformation power the core dynamo of Mars?, Icarus, 201, 31-43, doi:10.1016/j.icarus.2009.01.005.

Christensen, U. R., and J. Wicht (2007), Numerical dynamo simulations, in Treatise on Geophysics, vol. 8, edited by P. Olson, pp. 245-282, Elsevier, Amsterdam.

Frey, H. (2008), Ages of very large impact basins on Mars: Implications for the late heavy bombardment in the inner solar system, Geophys. Res. Lett., 35, L13203, doi:10.1029/2008GL033515.

Holsapple, K. A. (1993), The scaling of impact processes in planetary sciences, Annu. Rev. Earth Planet. Sci., 21, 333-373, doi:10.1146/annurev. ea.21.050193.002001.

Kuang, W., W. Jiang, and T. Wang (2008), Sudden termination of Martian dynamo?: Implications from subcritical dynamo simulations, Geophys. Res. Lett., 35, L14204, doi:10.1029/2008GL034183.

Levy, M. A., and H. J. S. Fernando (2002), Turbulent thermal convection in a rotating stratified fluid, J. Fluid Mech., 467, 19-40, doi:10.1017/ S0022112002001350.
Lillis, R. J., H. V. Frey, and M. Manga (2008), Rapid decrease in Martian crustal magnetization in the Noachian era: Implications for the dynamo and climate of early Mars, Geophys. Res. Lett., 35, L14203, doi:10.1029/ 2008GL034338.

Melosh, H. J. (1989), Impact Cratering: A Geologic Process, Oxford Univ. Press, New York.

Nimmo, F., and D. J. Stevenson (2000), Influence of early plate tectonics on the thermal evolution and magnetic field of Mars, J. Geophys. Res., 105, 11,969-11,979, doi:10.1029/1999JE001216.

Pierazzo, E., A. M. Vickery, and H. J. Melosh (1997), A reevaluation of impact melt production, Icarus, 127, 408-423, doi:10.1006/icar. 1997.5713.

Roberts, J. H., R. J. Lillis, and M. Manga (2009), Giant impacts on early Mars and the cessation of the Martian dynamo, J. Geophys. Res., 114 E04009, doi:10.1029/2008JE003287.

Stevenson, D. J. (1987), Limits on lateral density and velocity variations in the Earth's outer core, Geophys. J. R. Astron. Soc., 88, 311-319.

Watters, W. A., M. T. Zuber, and B. H. Hager (2009), Thermal perturbations caused by large impacts and consequences for mantle convection, J. Geophys. Res., 114, E02001, doi:10.1029/2007JE002964.

Williams, J.-P., and F. Nimmo (2004), Thermal evolution of the Martian core: Implications for an early dynamo, Geology, 32, 97-100, doi:10.1130/G19975.1.

Yoder, C. F., A. S. Konopliv, D. N. Yuan, E. M. Standish, and W. M. Folkner (2003), Fluid core size of Mars from detection of the solar tide, Science, 300, 299-303, doi:10.1126/science.1079645.

Young, R. E., and G. Schubert (1974), Temperatures inside Mars: Is the core liquid or solid?, Geophys. Res. Lett., 1, 157-160, doi:10.1029/ GL001i004p00157.

J. Arkani-Hamed, Department of Physics, University of Toronto, $60 \mathrm{St}$. George St., Toronto, ON M5S 1A7, Canada. (jafar@physics.utoronto.ca)

P. Olson, Earth and Planetary Sciences, Johns Hopkins University, 3400 North Charles St., Baltimore, MD 21218, USA. 